\title{
A rare cause of acute lower abdominal pain: Emphysematous cystitis
}

\author{
Tarkan Ergün ${ }^{1}$, Halil Olcay Eldem² ${ }^{2}$ Hatice Lakadamyalı ${ }^{1}$
}

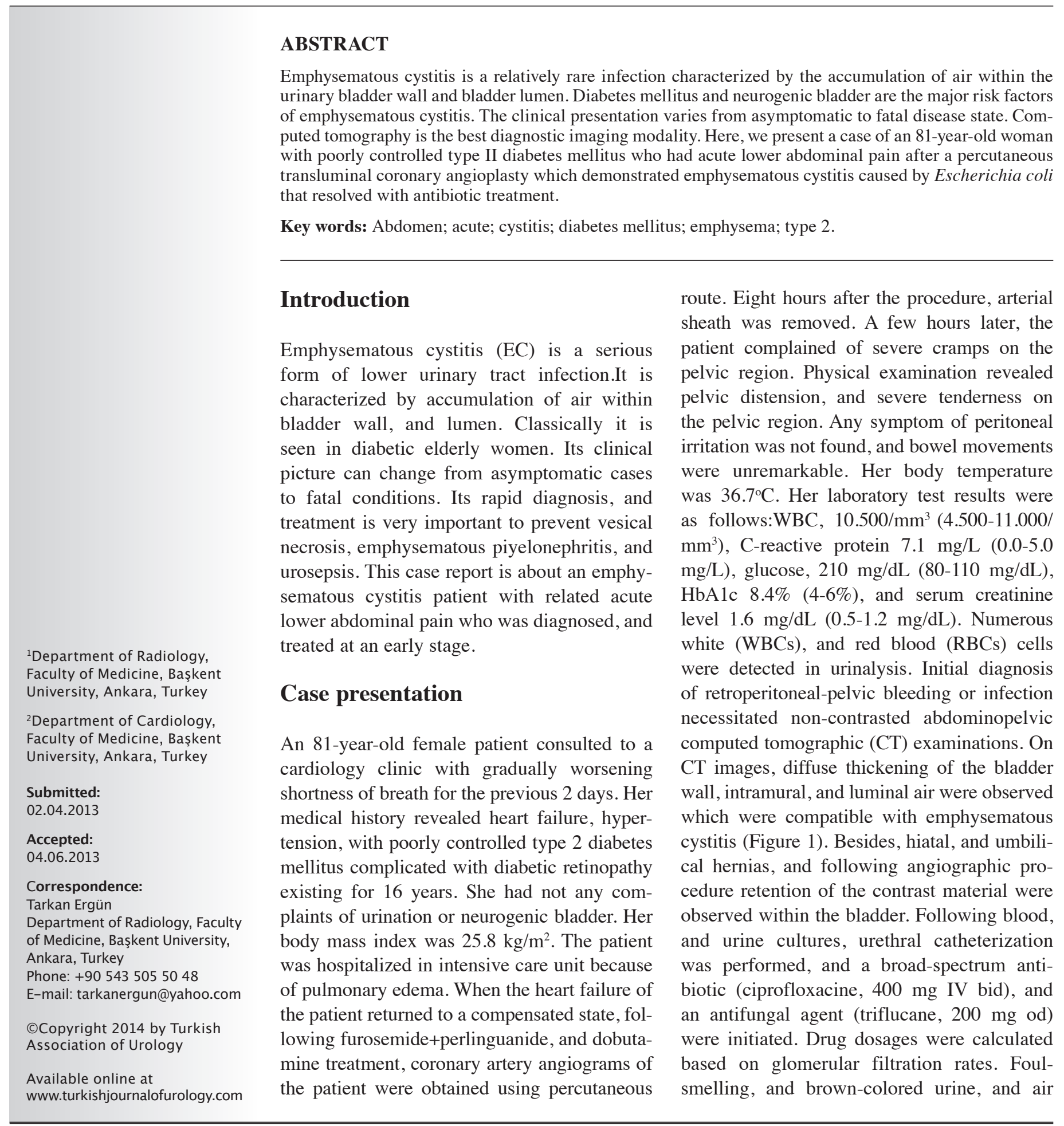




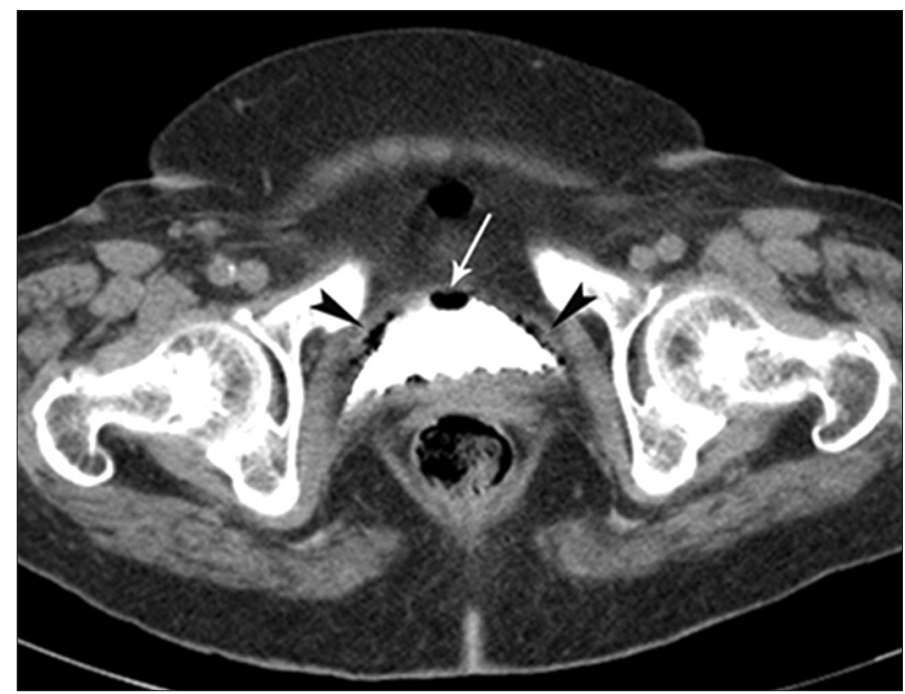

Figure 1. On non-contrasted CT section passing through the pelvic plane, and bladder wall (intramural) (black arrow), and extramural (white arrow) accumulation of air are observed

were observed in the drainage fluid of the bladder. Blood sugar was regulated with insulin. Two days later, clinical condition of the patient improved rapidly, her urine cleared, and laboratory test results gradually returned to normal. Escherichia coli $\left(10^{6}\right.$ CFU E. coli) susceptible to ciprofloxacine grew on urine culture media. Any bacterial growth was not detected on blood culture media. Triflucane therapy was stopped according to the culture results obtained. The patient received intravenous doses of antibiotherapy for a total of 5 days. Then, drainage catheter was withdrawn, a 14-day-oral ciprofloxacine (500 mg bid), and antidiabetic therapy were arranged before discharge of the patient. At the first month control visit, the patient was asymptomatic, and her laboratory test results were within normal limits.

\section{Discussion}

Emphysematous cystitis is a rarely seen infection of bladder caused by gas-forming bacteria. It was firstly described in 1671, when a patient complained of passage of gas from his urethra. Near the end of 1800s, for the first time, Eisenlohr detected intramural gas at autopsy. ${ }^{[1]}$ In the year 1961, this condition was defined as "emphysematous cystitis' by Bailey. ${ }^{[2]}$ Up to now more than 200 cases of EC have been cited. EC is generally seen in elderly women with diabetes mellitus. Recently, Toyota et al. ${ }^{[3]}$ evaluated 152 cases cited in the literature, and indicated that $63.4 \%$ of the cases were of female gender with a mean age of 69 years. Besides, diabetes mellitus (type I, 42.4\%, and type II $57.6 \%$ ) were detected in $66.7 \%$ of the cases. The effects of diabetes mellitus on urinary system include diabetic nephropathy, renal papillary necrosis, impairment of renal perfusion, and neuropathic bladder dysfunction. Together with these factors, glucosuria, and leucocytic dysfunction, and patients with diabetes mellitus are more prone to develop lower urinary tract infections. Besides, in these patients, complicated urinary system infections (renal, and perirenal abscess, fungal infections, xanthogranulomatous pyelonephritis, and infections caused by gas-producing microorganisms) are more frequently seen. ${ }^{[4]}$ Kuo et al. ${ }^{[5]}$ indicated that women are more prone to EC because of their increased predisposition to urinary tract infections.

The mechanism of gas formation in emphysematous cystitis has not been fully elucidated. The accepted mechanism is about accumulation of hydrogen, and carbon dioxide as a result of fermentation produced by infective organisms within tissues. In patients with diabetes mellitus, increased amounts of glucose in urine, and within tissues, and in non-diabetic patients albumin, and lactose have been thought to be the building blocks of gas production. ${ }^{[6]}$ Accumulation of gas as a result of fermentation increases local pressure within tissue, decreases tissue perfusion, and it might lead to tissue infarction at this level. Since infarcted tissue is a better culture media for gas-producing pathogens which also prevents gas transport, it results in the development of a worse vicious circle. Main risk factors for emphysematous cystitis consist of diabetes mellitus, urinary tract abnormalities, urinary stasis, and immune suppression. Besides, neurogenic bladder, bladder fistula, urethral catheterization, and recurrent urinary tract infections are also included among predisposing factors. In our case, EC developed following coronary angiography. However, any correlation between EC, and angiographic procedures has not been encountered in the literature.

Emphysematous cystitis has nonspecific clinical symptoms, Clinical condition may vary between asymptomatic disease or minor complaints of urinary tract infection to septic shock or peritonitis. Thomas et al.$^{[7]}$ reported that $7 \%$ of the cases cited in the literature were asymptomatic, and detected incidentally during abdominal imaging procedures. Kuo et al . ${ }^{[5]}$ indicated absence of any correlation between clinical symptoms, and severity of the inflammation Pain is the most frequently observed (80\%) symptom in emphysematous cytitis. In nearly $53 \%$ of the cases with urinary tract infections, classical symptoms can be observed. On physical examination mostly (65.6\%) abdominal tenderness is detected. While, peritoneal signs are seen only in $6.2 \%$ of the cases. Leukocyturia, and hematuria are observed in 87,5 , and $82.3 \%$ of the cases, respectively. ${ }^{[8]}$

In the diagnosis of emphysematous cytitis, a serious clinical symptom suggestive of the disease could not be found. The diagnosis is made radiographically. Direct radiography is a relatively sensitive diagnostic method. Since, radiographic signs can be confused with rectal gas, emphysematous vaginitis, pneumatosis intestinalis, and gas gangrene of uterus, this modality has a relatively lower specificity. Grupper et al. ${ }^{[8]}$ reported that only $11.3 \%$ of their EC cases could be diagnosed correctly using radiographic means. The most reliable diagnostic imaaging modality is CT. ${ }^{[9]}$ CT clearly reveals severity, and extent of the disease (presence 
of an associated ascending infection). It ensures discrimination among urinary pathologies communicating with the outer environment, and hence air (urointestinal fistula, tissue infarct associated with necrosis, trauma, and instrumentation) In addition, it demonstrates enterovesical fistula which might develop in association with EC. In their case series Grupper et al. ${ }^{[8]}$ reported gas formation within the bladder wall, and its lumen in 94.4 , and $3.7 \%$ of their cases, respectively. In our case gas formation was observed both within the bladder wall, and vesical lumen. Because of difficulties encountered in the evaluation of ultrasonographic, and magnetic resonance images, these imaging modalities have relatively lower degrees of sensitivity. However cystoscopy is not adequate as a single diagnostic modality for EC. However it can evaluate the presence of bladder outlet obstruction. On histopathological examination, induration, and gas filled vesicles are observed within the vesical wall. On microscopic examination especially on the bladder mucosa, gas filled vesicles, and surrounding fibrocytes, and multinuclear giant cells are seen.

Many gas-forming microorganisms can cause EC. The most frequently isolated microorganisms in urine cultures in decreasing order of frequency have been E. coli (57.1\%), klebsiellae (21.8\%), and enterococci $(6.8 \%) .{ }^{[3]}$ In our urine culture E. coli was detected.

Treatment of the emphysematous cystitis is dependent on the severity of the disease. Generally treatment consists of administration of broad-spectrum antibiotics, bladder drainage, and correction of the underlying predisposing disease. In addition, because of its unfavourable contribution to gas production, glycemic control is extremely important. As is in our case, antibiotherapy should be admin,stered through an intravenous route. However, Thomas et al. proved that $9 \%$ of the cases reported in the literature had been treated only with oral antibiotics. ${ }^{[7]}$ In most of the cases, treatment is initiated with broad-spectrum antibiotics. Based on the pathogenic agent isolated from culture, more specific antibiotic(s) can be used instead. A consensus has not been reached about the duration of the treatment. Grupper et al. ${ }^{[8]}$ reported average duration of treatment, and hospitalization as 10 , and 7 days, respectively. In serious cases or those refractory to conservative treatment, surgical treatment (partial cystectomy, cystectomy, and surgical debridement) is required Thomas et al. ${ }^{[7]}$ reported that they had treated $90 \%$ of their 135 cases with medical therapy, and the remainder $(10 \%)$ of their patients had required surgical intervention. While, $15 \%$ the EC cases in Grupper et al.$^{[8]}$ series required laparotomy.

Generally emphysematous cystitis has a benign course. However in $19 \%$ of their cases complications might develop. In their series, Grupper et al. ${ }^{[8]}$ detected higher complication rates in cases with diabetes mellitus, immune suppression or urinary system pathology relative to those without. Besides, they published higher mortality rate $(9.4 \%)$ for their AC series. However, Thomas et al. ${ }^{[7]}$ reported mortality rates of 7 , and $14 \%$ in cases confined only to bladder, and in patients with associ- ated emphysematous infection on another extravesical location of the urinary system, respectively. Therefore presence of an emphysematous infection on other site of the urinary system is accepted as an important prognostic factor for EC.

In conclusion, clinicians should kept this clinical entity in mind in the differential diagnosis of acute lower abdominal pain in especially high-risk patients. Early diagnosis, and treatment of EC are very important issues, since it progresses rapidly leading to bladder necrosis, emphysematous pyelitis, urosepsis, and finally death. Treatment generally consists of administration of broad-spectrum antibiotics, adequate urine drainage, and glycemic control. EC usually has an excellent prognosis.

Informed Consent: Written informed consent was obtained from patients who participated in this case.

Peer-review: Externally peer-reviewed.

Author Contributions: Concept - T.E.; Design - T.E., H.O.E.; Supervision - T.E., H.L.; Funding - H.L.; Materials - H.O.E.; Data Collection and/or Processing - T.E.; Analysis and/or Interpretation T.E.; Literature Review - T.E.; Writer - T.E.; Critical Review - T.E., H.L., H.O.E.; Other - T.E.

Conflict of Interest: No conflict of interest was declared by the authors.

Financial Disclosure: The authors declared that this study has received no financial support.

\section{References}

1. Eisenlohr W. Interstitial vaginal, intestinal, and bladder emphysema caused by gas-forming bacteria. Beitr Path Anat Allg Path 1888;3:101.

2. Bailey H. Cystitis emphysematosa; 19 cases with intraluminal and interstitial collections of gas. Am J Roentgenol Radium Ther Nucl Med 1961;86:850-62.

3. Toyota N, Ogawa D, Ishii K, Hirata K, Wada J, Shikata K, et al. Emphysematous cystitis in a patient with type 2 diabetes mellitus. Acta Med Okayama 2011;65:129-33.

4. Akan O. Rare infections in diabetes. Turkiye Klinikleri J EndocrinSpecial Topics 2008;1:64-70.

5. Kuo CY, Lin CY, Chen TC, Lin WR, Lu PL, Tsai JJ, et al. Clinical features and prognostic factors of emphysematous urinary tract infection. J Microbiol Immunol Infect 2009;42:393-400.

6. Yang WH, Shen NC. Gas-forming infection of the urinary tract: an investigation of fermentation as a mechanism J Urol 1990;143:960-4.

7. Thomas AA, Lane BR, Thomas AZ, Remer EM, Campbell SC, Shoskes DA. Emphysematous cystitis: a review of 135 cases. BJU Int 2007;100:17-20.

8. Grupper M, Kravtsov A, Potasman I. Emphysematous cystitis: illustrative case report and review of the literature. Medicine (Baltimore) 2007;86:47-53.

9. Sarısoy HT, Gürcan NI, Demirci A. Radiologic imaging in urinary tract infections. Turkiye Klinikleri J Urology-Special Topics 2010;3:19-26. 